\title{
Die neue Redaktion stellt sich vor
}

Von unserem Kollegen und Freund Th. Abelin übernehmen wir eine verantwortungsvolle Aufgabe. Wir werden versuchen, ihr gerecht zu werden. Unter der Redaktion von Th. Abelin waren die wissenschaftlichen und praktischen Belange der Sozial- und Präventivmedizin im gleichen Masse berücksichtigt. So brachte die Zeitschrift Beiträge, welche die wissenschaftlichen Grundlagen des Arbeitsgebietes untermauerten, und sie wies Wege, die Theorien und Prinzipien in die Praxis und Förderung unseres Faches umzusetzen. Die vorbildliche Tradition, welche uns hinterlassen wurde, möchten wir nach bestem Können weiterführen. Die bisherige Richtung wird im wesentlichen beibehalten. Insbesondere betrachten wir Themenhefte als wichtig und unerlässlich, denn nur in solchen kann ein bestimmtes, umschriebenes Gebiet in all seinen Zusammenhängen übersichtlich und organisch dargestellt werden. Wir möchten jedoch auch jeweils freie Beiträge in den Themenheften unterbringen, um die Wartezeit für aktuelle Artikel zu verkürzen. Die «freie Nummer» wird indessen weitergeführt.

Die Sozial- und Präventivmedizin, wie auch die Epidemiologie, sind in vieler Hinsicht Grenzgebiete. Unsere Beziehungen zu den Soziologen und Wirtschaftsforschern haben sich ausgeweitet. Der Wichtigkeit psychologischer Aspekte wird Rechnung getragen. Die gegenseitigen Beziehungen zur klinischen Medizin lassen, wie überall auf der Welt, noch zu wünschen übrig. Wir möchten unser möglichstes tun, all diese Wechselwirkungen und Zusammenarbeit aufrechtzuerhalten und zu fördern.

Es ist unsere Absicht, die Redaktion als eine Art «Triumvirat» zu führen. Aus administrativen Gründen ist ein Chefredaktor erforderlich, und er muss schliesslich die Verantwortung tragen. Der Leser soll jedoch wissen, dass wir alle drei an der Arbeit beteiligt sind. Wir danken für das Vertrauen unserer Leser sowie das wegweisende Beispiel von Th. Abelin und seine grosse Hilfe bei der Übergabe der Redaktion.

R. Bruppacher

F. H. Epstein

J. Martin

\section{La nouvelle Rédaction se présente}

Nous reprenons de notre collègue et ami Th. Abelin une tâche pleine de responsabilités et nous essaierons d'en être dignes. Pendant le mandat rédactionnel du professeur Abelin, les préoccupations scientifiques et pratiques de la médecine sociale et préventive ont bénéficié de la même attention. Ainsi, notre revue a publié des contributions qui étayaient les fondements scientifiques de leur domaine d'investigation. Dans d'autres communications, elle a aussi présenté de nouvelles façons de traduire dans la pratique et la recherche les théories et les principes de notre discipline. Nous souhaitons poursuivre, au mieux de nos capacités, la tradition exemplaire qui nous est confiée et garder pour l'essentiel la même orientation. En particulier, nous considérons indispensable de conserver le système des numéros à thème, qui nous paraissent être le meilleur moyen de présenter un domaine donné de manière complète, dans ses composantes et dans ses interrelations. Nous voulons cependant accueillir des communications libres aussi dans les numéros à thème, de façon à raccourcir le délai d'attente pour des articles d'actualité. Le «numéro libre» annuel (sans thème fixé) sera aussi maintenu.

La médecine sociale et préventive, comme d'ailleurs l'épidémiologie, sont à divers égards des domaines«frontière». Nos relations avec les sociologues et les économistes se sont beaucoup étoffées. Il s'agit aussi de donner l'attention voulue à l'importance des aspects psychologiques. Nos relations mutuelles avec la médecine clinique cependant, comme partout dans le monde, laissent encore à désirer. Nous ferons de notre mieux pour maintenir, et autant que possible promouvoir, dans le futur, ces échanges et collaborations.

Notre intention est de conduire le travail de la Rédaction comme une sorte de triumvirat. Pour des raisons administratives, il doit y avoir un rédacteur en chef, qui assume la responsabilité ultime de l'entreprise. Sachez cependant que nous sommes tous trois impliqués dans la marche de la revue et le labeur correspondant. Nous remercions les lecteurs de Médecine sociale et préventive de leur confiance et tenons à redire notre gratitude à Th. Abelin pour son exemple et pour son aide précieuse lors de la remise de la Rédaction.

R. Bruppacher

F. H. Epstein

J. Martin 